\title{
APPLICATION OF CdZnTe QUASI- HEMISPHERICAL DETECTORS IN STRONG GAMMA-RADIATION FIELDS
}

\author{
Anatoli Loutchanski \\ ZRF RITEC SIA \\ Riga, Latvia \\ a.loutchanski@ritec.lv \\ Victor Ivanov \\ ZRF RITEC SIA \\ Riga, Latvia \\ v.ivanov@ritec.lv
}

\author{
Viktors Fjodorovs \\ ZRF RITEC SIA \\ Riga, Latvia \\ v.fjodorof@ritec.lv \\ Vadims Ogorodniks \\ ZRF RITEC SIA \\ Riga, Latvia \\ v.ogorodnik@ritec.lv
}

\begin{abstract}
The results of a study of some ways to improve spectroscopy characteristics of the CdZnTe quasihemispherical detectors when working in high gamma radiation fluxes are presented. It was shown that the use of IR illumination with a wavelength of $1050 \mathrm{~nm}$ or $1200 \mathrm{~nm}$ or at slight warm-up of the detector to $+30^{\circ} \mathrm{C} \ldots+40^{\circ} \mathrm{C}$ can significantly improve spectroscopy performance of the $\mathrm{CdZnTe}$ detectors of size $3.5 \mathrm{~mm} \times 3.5 \mathrm{~mm} \times 1.75 \mathrm{~mm}$ when operating in a tested gamma-radiation field with a dose rate up to $590 \mathrm{mGy} / \mathrm{h}$.
\end{abstract}

Keywords-CdZnTe (CZT), gamma-radiation detectors, semiconductor radiation detectors, room temperature semiconductor detectors, infra-red (IR) illumination, IR LED, high flux gamma-radiation, leakage current, voltage-current characteristic

\section{INTRODUCTION}

Presently room temperature CdZnTe (CZT) nuclear radiation detectors of various designs and sizes are widely used for spectrometric measurements of X-and gammaradiations. In most cases, the spectrometric detectors with highest efficiency are required. However, there are tasks where good spectroscopic CZT detectors with a low efficiency should be used. These tasks are connected with spectrometric measurements in high gamma-radiation fluxes. Small size quasi-hemispherical CZT detectors [1-6] are already successfully used to control nuclear spent fuel or/and highlevel radiation waste. Recently a new system for verification of nuclear spent fuel assemblies using Passive GammaEmission Tomography (PGET) [7] was developed and tested. The system contains about 200 detector modules with CZT quasi-hemispherical detectors of size $3.5 \mathrm{~mm} \times 3.5 \mathrm{~mm} \times$ $1.75 \mathrm{~mm}$. More than 1000 such detectors were tested. Approximately, only half of the measured detectors successfully passed tests under high gamma-radiation fluxes and were found suitable for subsequent use in the PGET system. Therefore, study of causes of the detectors' characteristics deterioration in high gamma-radiation fluxes and search for ways to eliminate these causes is an urgent task. It was previously shown [8] that IR illumination with infrared light with wavelengths close to the absorption edge of the $\mathrm{CdZnTe}$ can significantly improve the performance of the quasi-hemispherical detectors operating at room and low temperatures. In this work, we studied the effects of IR illumination and heating of the quasi-hemispherical detectors operating in high gamma-radiation fluxes from the ${ }^{137} \mathrm{Cs}$.

\section{RESULTS AND DISCUSSION}

For measurements, experimental setup based on application of a calibrated measurement bench with a high activity gamma-radiation source ${ }^{137} \mathrm{Cs}$ was used. The stand allows changing distance between the source and the detector. The maximum achievable dose rate at the measurement point with the minimal distance between the source and the detector $(\mathrm{R}=28 \mathrm{~cm})$ was about $590 \mathrm{mGy} / \mathrm{h}$. Detector fixture allowing installation and quick change of the detectors and IR LED. CZT quasi-hemispherical detectors of size $3.5 \mathrm{~mm} \times 3.5 \mathrm{~mm}$ $\times 1.75 \mathrm{~mm}$ fabricated of different raw CdZnTe crystals were tested. Changeable IR LED with wavelengths $940 \mathrm{~nm}$, $1050 \mathrm{~nm}$ and $1200 \mathrm{~nm}$ were used at measurements. Used heater allows heating the detector up to the $+50^{\circ} \mathrm{C}$. During the measurements LED forward current, heater consumption currents and operation temperature were controlled. Detector's leakage currents and spectra of ${ }^{137} \mathrm{Cs}$ were recorded. Schematic experimental setup is shown on Fig. 1.

Intense gamma irradiation significantly affects leakage currents and spectrometric characteristics of the CZT detectors. Fig. 2 show typical voltage-current characteristics of CZT quasi-hemispherical detectors measured at room

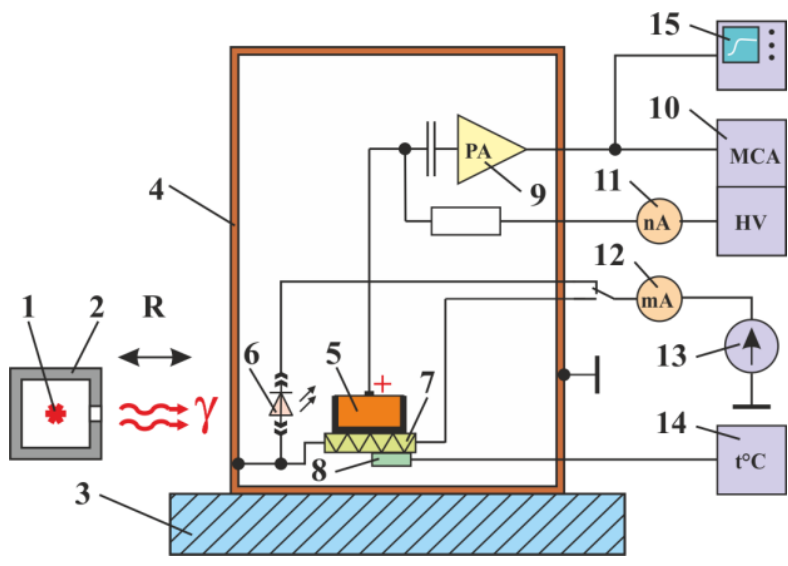

Fig. 1. Schematic experimental setup.

1-highly active radiation source ${ }^{137} \mathrm{Cs}$; 2 -lead container/collimator; 3 movable stand; 4-detector fixture; 5-CZT detector; 6-IR LED; 7-heater; 8-temperature sensor; 9-charge sensitive preamplifier; 10-digital multichannel analyzer type MCA527 with high voltage power supply; 11-detector leakage current meter (resolution $1 \mathrm{nA}$ ); 12-LED forward current or heater consumption current meter; 13-stabilized current source for IR LED or heater; 14-temperature monitor; 15-digital oscilloscope. 


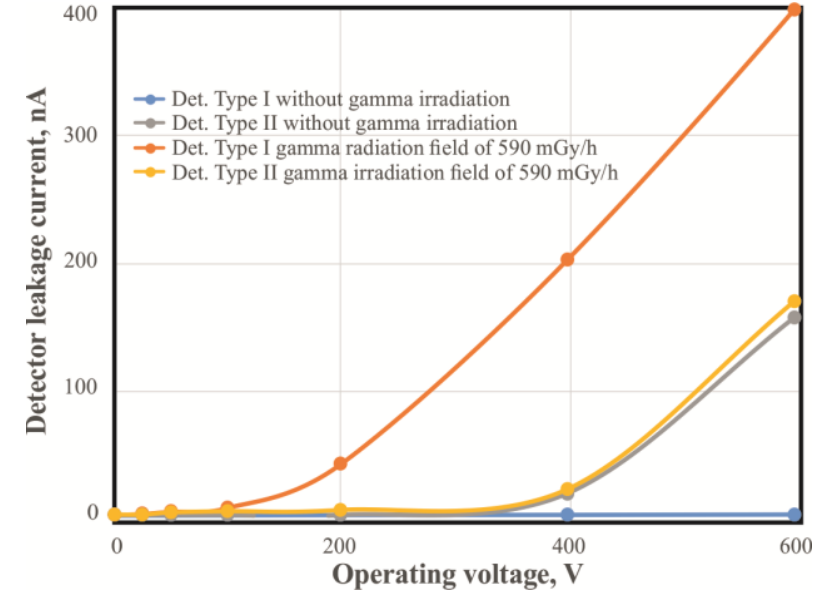

Fig. 2. Voltage-current characteristics of CZT quasi-hemispherical detectors measured at room temperature without and under influence of high gamma-radiation fluxes.

temperature without and in high gamma-radiation flux. Processing of measurement results allows distinguishing two types of detectors differing in a behavior of leakage currents under gamma irradiation. Type I - are detectors that have a significant (20 .. 100 times) increase in leakage current under influence of strong gamma irradiation. Large increasing of the leakage currents were accompanied by a significant deterioration or even complete disappearance of the spectrometric capabilities of such detectors. These detectors, as a rule, had insignificant leakage currents $<5 \mathrm{nA}$ at room temperature at $400 \mathrm{~V}$ without exposure to intense gamma irradiation. Type II - are detectors that did not have or had a slight increase in leakage current under to influence of gamma irradiation. These detectors, as a rule, had significant leakage currents $20 \ldots 300 \mathrm{nA}$ at $400 \mathrm{~V}$ without exposure to intense irradiation and did not allow increasing the operating high voltage to acceptable values due to a significant increase in the noise level with increasing voltages.

For detectors of the first type, operating in high gammaradiation fluxes, complex dependencies of the leakage currents on IR illumination intensity with IR LED wavelength of $1050 \mathrm{~nm}$ were obtained, Fig. 3 in the range of the LED operating currents $(\leqslant 100 \mathrm{~mA})$, the LED relative radiant intensity is approximately directly proportional to the LED forward current. With increasing of the LED forward current,

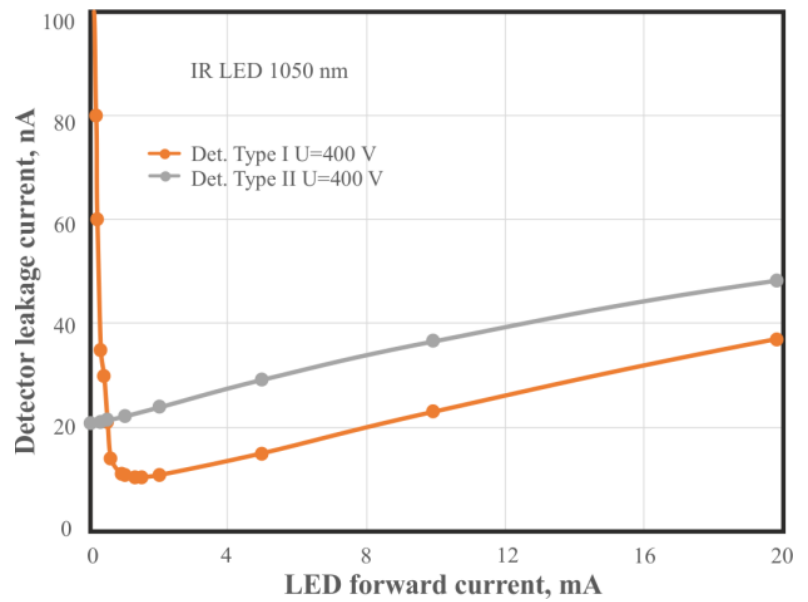

Fig. 3. Detectors leakage currents versus IR LED $(1050 \mathrm{~nm})$ forward current measured in gamma-radiation field of $590 \mathrm{mGy} / \mathrm{h}$.

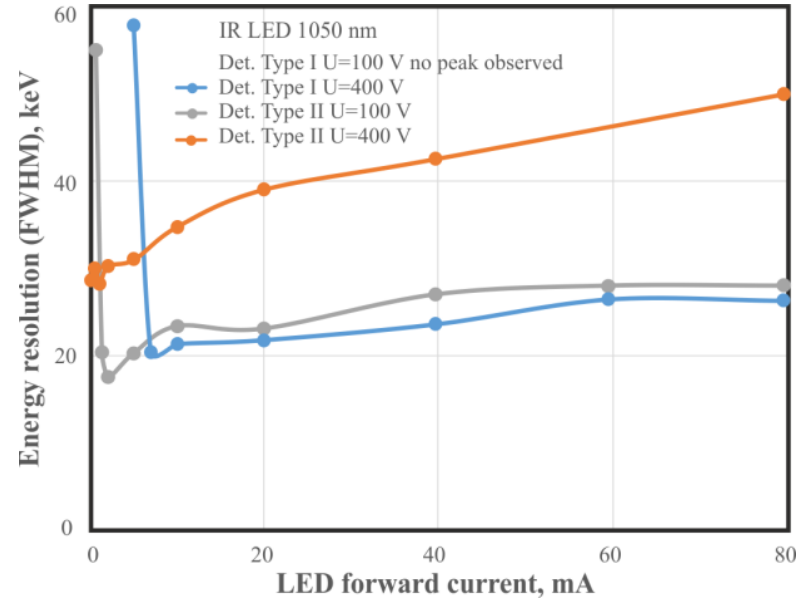

Fig. 4. Energy resolution (FWHM) at $662 \mathrm{keV}$ versus IR LED forward current at different operating voltages measured in gamma-radiation field of $590 \mathrm{mGv} / \mathrm{h}$.

the leakage currents of such detectors first decreases, then reaches the minimum, and then begins to rise slowly. The leakage current of the second type detectors increases with rising of the LED forward current.

The behavior of the energy resolution of the detectors of both types under IR illumination also had own peculiarities, Fig. 4. First, it improves noticeably with increasing of IR LED forward current and then starts to deteriorate. Moreover, such a dependence of energy resolution on values of the LED direct current for detectors of the second-type was observed only at relatively low operating voltages $<200 \mathrm{~V}$. At higher operating voltages, the energy resolution of the second-type detectors deteriorated with increasing of IR illumination intensity.

Illumination by IR light with wavelengths of $1050 \mathrm{~nm}$ or $1200 \mathrm{~nm}$ as well was found to greatly improve spectrometric characteristics of the quasi-hemispherical CZT detectors operating in high gamma-radiation fluxes. This allows expanding the range of count rates in which the normal operation of the detectors is observed. Fig. 5 demonstrates dependence of energy resolution versus count rate obtained with different levels of IR illumination. Count rate was varied by changing the distance between radioactive source and tested detector. Illumination by IR light with wavelengths of $940 \mathrm{~nm}$ did not produce positive effect.

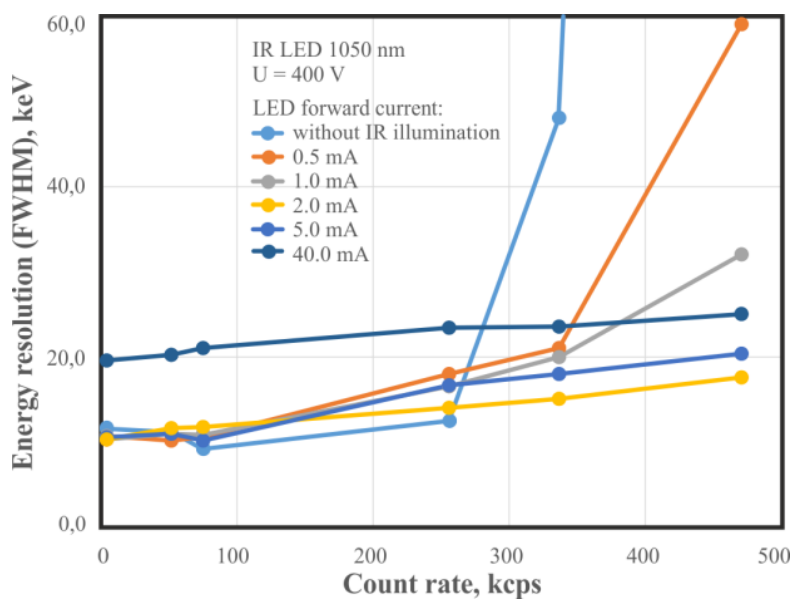

Fig. 5. Energy resolution (FWHM) at $662 \mathrm{keV}$ versus recorded count rate measured without and with IR LED illumination with different LED forward currents. 


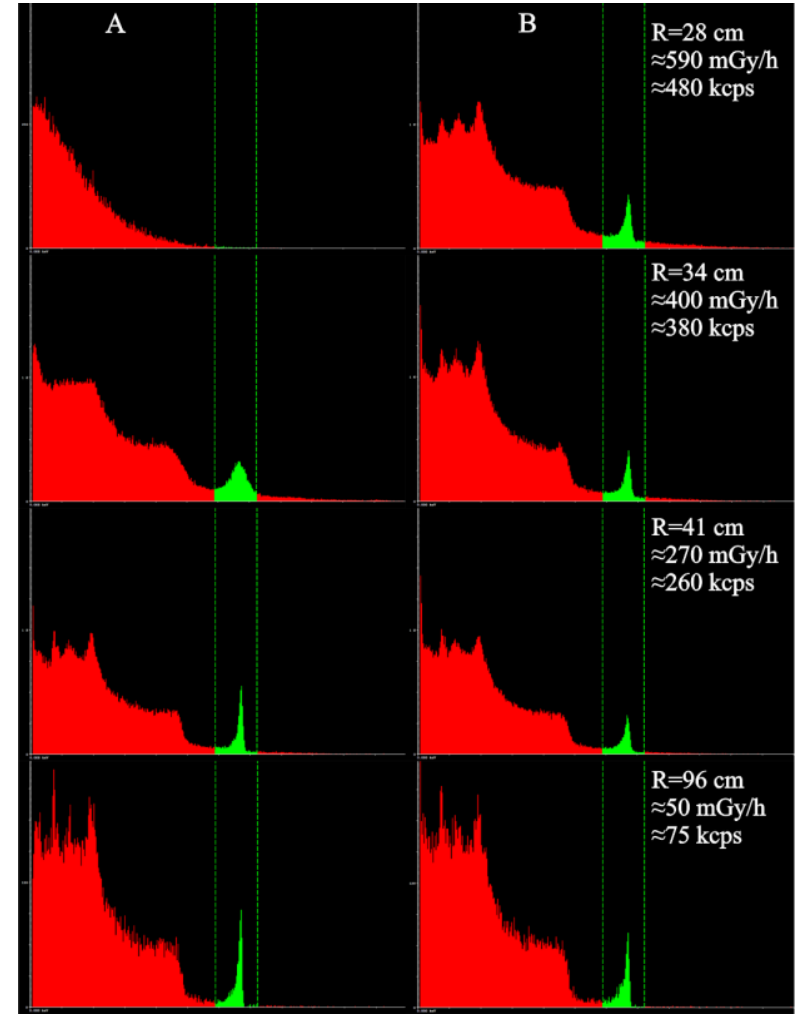

Fig. 6. Spectra of ${ }^{137} \mathrm{Cs}$ measured without (A) and with (B) IR illumination at different distances between radiation source and detector.

Fig. 6 illustrates changes in the shape of ${ }^{137} \mathrm{Cs}$ spectra measured at different distances between the radiation source and the detector without and with IR illumination $(1050 \mathrm{~nm})$ The LED forward current $2 \mathrm{~mA}$ and detector operating voltage $400 \mathrm{~V}$ were applied.

A study of temperature dependence of the detector's leakage currents and energy resolutions of the detectors operating in a high gamma radiation fluxes was carried out. A decrease (quenching) of the detector's leakage currents and energy resolution improvement with a slight increase in the operating temperature by $+5^{\circ} \mathrm{C} \ldots+10^{\circ} \mathrm{C}$ were found, Fig. 7 dependences were measured in gamma-radiation field of $590 \mathrm{mGy} / \mathrm{h}$ without IR illumination.

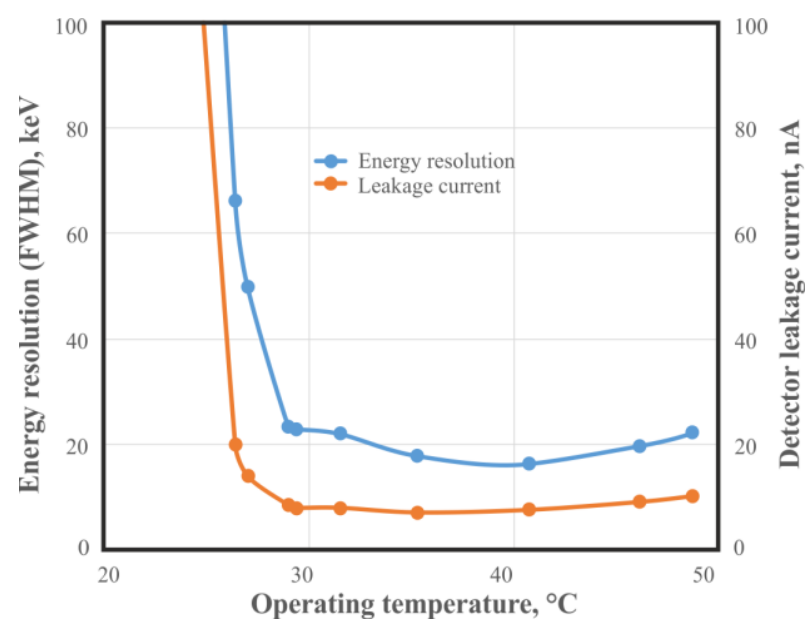

Fig. 7. Temperature dependencies of energy resolution (FWHM) at $662 \mathrm{keV}$ and leakage current at $400 \mathrm{~V}$.

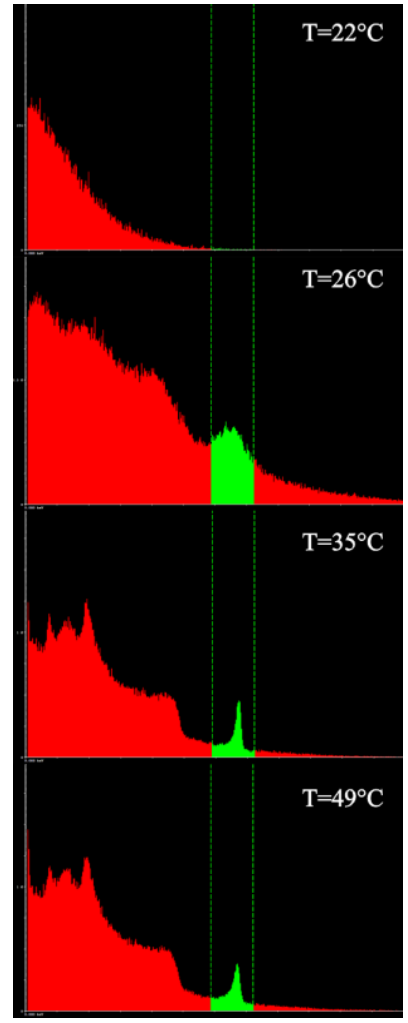

Fig. 8. Spectra of ${ }^{137} \mathrm{Cs}$ measured without IR illumination at different operating temperatures in radiation field of $590 \mathrm{mGy} / \mathrm{h}$. Operating voltage $400 \mathrm{~V}$.

The Table I shows the measurement results of energy resolutions of two types CZT quasi-hemispherical detectors of $3.5 \mathrm{~mm} \times 3.5 \mathrm{~mm} \times 1.75 \mathrm{~mm}$ without and with IR illumination measured in a weak and in a high gamma-radiation flux.

TABLE I.

\begin{tabular}{|c|c|c|c|c|c|}
\hline \multirow[b]{3}{*}{ 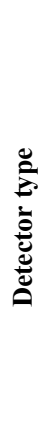 } & \multirow{3}{*}{ 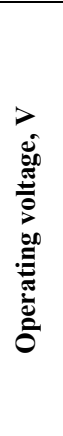 } & \multicolumn{4}{|c|}{ Energy resolution (FWHM) at $662 \mathrm{keV}, \mathrm{keV}$} \\
\hline & & \multirow{2}{*}{$\begin{array}{c}\begin{array}{c}\text { Count rate } \\
<5 \text { kcps }\end{array} \\
\\
\\
\end{array}$} & \multicolumn{3}{|c|}{$\begin{array}{l}\text { Count rate } \\
\approx 500 \mathrm{kcps}\end{array}$} \\
\hline & & & 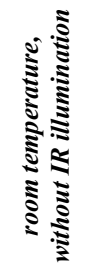 & 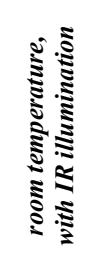 & 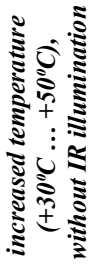 \\
\hline \multirow{5}{*}{ I } & 50 & 19 & no peak & no peak & 58 \\
\hline & 100 & 15 & no peak & no peak & 15 \\
\hline & 200 & 13 & no peak & 14 & 13 \\
\hline & 400 & 12 & no peak & 18 & 16 \\
\hline & 600 & 10 & no peak & 24 & 17 \\
\hline \multirow{5}{*}{ II } & 50 & 16 & no peak & 26 & 24 \\
\hline & 100 & 15 & no peak & 24 & 28 \\
\hline & 200 & 13 & 28 & 25 & 23 \\
\hline & 400 & 16 & 44 & 37 & 52 \\
\hline & 600 & 29 & 62 & 57 & 61 \\
\hline
\end{tabular}

\section{MAIN RESULTS}

Operating in high gamma-radiation fluxes can significantly worsen spectroscopy performance of the tested quasi-hemispherical detectors. 
Tested detectors can be divided into two groups. The first are detectors with rather low leakage currents $<5 \mathrm{nA}$ at $400 \mathrm{~V}$ without gamma-irradiation but with strongly increasing leakage currents (to $20 \ldots 100$ times) under influence of the high intensity gamma-radiation flux. In second group are detectors with leakage currents of about $20 \ldots 300 \mathrm{nA}$ at $400 \mathrm{~V}$ without gamma-irradiation, which only slightly increasing in high gamma-radiation flux.

Illumination by IR light with wavelengths of $1050 \mathrm{~nm}$ or $1200 \mathrm{~nm}$ as well as heating up to $+30^{\circ} \mathrm{C} \ldots+40^{\circ} \mathrm{C}$ was found to greatly improve spectrometric characteristics of the quasihemispherical CZT detectors operating in high gammaradiation fluxes.

For detectors of the first type, complex dependencies of the leakage currents and the energy resolutions on forward currents of the IR LED and operating temperatures were obtained. With increasing IR illumination intensity or with increasing operating temperature, the leakage current of such detectors first decreases, reaches the minimum, and then begins to rise slowly. The behavior of the energy resolution is similar - first it improves noticeably and then starts to deteriorate.

Such complex behavior of leakage currents and energy resolution under the influence of high gamma-radiation fluxes and IR illumination can be associated with a change in an electric field distribution in the detector caused by formation of a space charge due to trapping of photo generated holes at deep levels and their detrapping under the influence of IR illumination [9]. A detailed explanation of the effects found requires additional research.

\section{REFERENCES}

[1] K. Zanio, "Use of Various Device Geometries to Improve the Performance of CdTe Detectors," Rev. de Phys. Appl., vol. 12, pp. 343347, 1977.

[2] L. A. Alekseeva, P. G. Dorogov, V. I. Ivanov, A. K. K husainov, "Cadmium telluride gamma radiation detectors with a high energy resolution," Pribory i Teknika Eksperimentya, no. 1, pp. 54-58, 1985.

[3] V. Ivanov, P. Popov, A. Loutchansky, L. Aleksejeva, E. Mozchaev, "Further Development of emispherical CdZnTe Detectors for Safeguards Applications," Proc. 21th Annual ESARDA Symp. on Safeguards and Nuclear Material Management, Seville, Spain, pp. 479 $-484,1999$.

[4] C. Szeles, D. Bale, J, Grosholz, G. L. Smith, M. Blostein, J. Eger, "Fabrication of High Performance CdZnTe Quasi-Hemispherical Gamma-ray CAPuretm Plus Detectors," Hard X -Ray and Gamma-Ray Detector Physics VIII, Proceedings of SPIE, Vol. 6319 , (SPIE, Bellingham, W A, 2006

[5] V. Ivanov, P. Dorogov, A. Loutchansky, and L. Aleksejeva, "CZT quasi-hemispherical detectors with improved spectrometric characteristics," in Proc. IEEE NSS-MIC Conf. Rec., Orlando, FL, Oct. 25-31, 2009.

[6] Spectrometric detection probes Types SDP310 and SDP500, ZRF RITEC SIA Data Sheets, http://www.ritec.lv.

[7] T. White, M. Mayorov, A. Lebrun, P. Peura, T. Honkamaa, J. Dahlberg, J. Keubler, V. Ivanov, A. Turenen, "Application of Passive Gamma Emission Tomography (PGET) for the Verification of Spent Nuclear Fuel", INMM 59th Annual Meeting, Baltimore, Maryland, USA, July 22-26, 2018

[8] V. Ivanov, P. Dorogov, A. Loutchansky, L. Grigorjeva, D. Millers, "Improving the Performance of Quasi-Hemispherical CdZnTe Detectors Using Infrared Stimulation", IEEE Trans. Nucl. Sci., vol. 59, no. 5 , pp. 2375-2382, 2012.

[9] Jakub Pekárek, Václav Dědič, Jan Franc, Eduard Balas, Martin Rejhon, Pavel Moravec, Jan Touš, Josef Voltr, "Infrared LED Enhanced Spectroscopic CdZnTe Detector Working under High Fluxes of Xrays", Sensors 2016, 16(10), 1591. 